Check for updates

Cite this: RSC Adv., 2017, 7, 39726

Received 19th May 2017

Accepted 4th August 2017

DOI: $10.1039 / \mathrm{c7ra05637k}$

rsc.li/rsc-advances

\section{Estimating contaminant source in chemical industry park using UAV-based monitoring platform, artificial neural network and atmospheric dispersion simulation}

\author{
Sihang Qiu, (D) a Bin Chen, (D) *a Rongxiao Wang, ${ }^{\text {a }}$ Zhengqiu Zhu, ${ }^{a}$ Yuan Wang ${ }^{b}$ \\ and Xiaogang $\mathrm{Qiu}^{\mathrm{a}}$
}

Airborne contaminants emitted from chemical industry parks can pose a potential threat to the environment. Therefore, using the data obtained from concentration-monitoring of the contaminant to find the source is of high importance. Most previous source estimation methods collect meteorological parameters and concentration measurements from static sensors. However, some meteorological parameters such as atmospheric stability and cloud cover are difficult to measure precisely. Furthermore, installing only several static sensors does not provide enough sampling data. In this paper, a novel approach is proposed to find the location of an emission source as well as its release rate in a chemical industry park. An unmanned aerial vehicle (UAV) monitoring platform is applied to sample sufficient and high-quality concentration data. Afterwards, an artificial neural network (ANN) trained by an atmospheric dispersion simulation tool is used to locate and quantify the emission source from candidate solutions, bypassing data on the atmospheric stability and other hard-to-obtain meteorological parameters. A numerical simulation with different conditions is implemented to test the accuracy and stability of the proposed approach. A real experiment is conducted in Shanghai to test the performance and sensitivity of this approach as well as the robustness of the monitoring platform. The results show that the approach proposed in this paper can effectively estimate the contaminant source in chemical industry parks. Both the numerical and real experiments prove that the proposed method is less sensitive to errors in meteorological data and concentration measurements than traditional source estimation methods including Bayesian inference and optimization.

\section{Introduction}

With industrial development, airborne contaminants have seriously influenced the health of human beings. Monitoring industrial emissions has therefore become an important issue. Locating and quantifying contaminant sources is one of the essential tasks of air contaminant monitoring and chemical industry administration. ${ }^{1-5}$ Generally, in order to support accurate source estimation, a large number of monitoring stations should be established to sample sufficient highquality monitoring data. ${ }^{6}$ Doubtlessly, building too many monitoring stations can be prohibitively expensive, and it is also difficult to manage a large number of stations. To address this challenge, we have developed an approach based on an unmanned aerial vehicle (UAV) monitoring platform, an

${ }^{a}$ College of Information System and Management, National University of Defense Technology, Changsha 410073, China.E-mail: nudtcb9372@gmail.com

${ }^{b}$ College of Territorial Resources and Tourism, Anhui Normal University, Wuhu 241003, China artificial neural network (ANN) and an atmospheric dispersion simulation tool to find the optimal (most likely) atmospheric dispersion source.

Generally, measurements of contaminants are obtained by static monitoring stations. However, static monitoring stations are usually not densely distributed enough to sample highquality data containing useful information from the contaminant plume. In this case, it is difficult to estimate the emission source from the data collected from these monitoring stations even when they are equipped with high resolution sensors. As a result, researchers have attempted to use aircraft to obtain high-quality air concentration measurements of contaminants. ${ }^{6-14}$ For example, an aircraft was used for monitoring nitrogen emissions from point sources. ${ }^{9}$ A previous study also demonstrated a method of source estimation using remote aircraft. ${ }^{8}$ In addition, White et al. proposed a monitoring network based on UAV sensors. ${ }^{12,13}$ Sanada and Torii analyzed the radioactive pollutant concentration via an unmanned helicopter after the Fukushima Dai-ichi nuclear power plant accident. ${ }^{11}$ UAV platforms can also be applied for predicting the 
$\mathrm{PM}_{2.5}$ concentration, ${ }^{14}$ contaminant plumes and volcanic plumes. ${ }^{7,10}$ The use of UAV platforms therefore allows many problems in traditional monitoring methods using static sensors to be addressed.

An important application of concentration measurements is using them to locate and quantify the dispersion source of contaminants. In the past few years, many methods and tools have been used to find contaminant sources. ${ }^{15}$ Most estimation methods are based on Bayesian inference or optimization. ${ }^{6}$ With Bayesian inference, the dispersion source can be estimated by calculating the posterior function. The Markov Chain Monte Carlo (MCMC) algorithm is a useful tool for posterior function calculation and source estimation., ${ }^{3,8,16-18}$ Some advanced filter or optimization methods such as particle filter, ${ }^{19-21} \mathrm{EnKF},{ }^{22}$ and $\mathrm{PSO}^{23,24}$ are also widely used in source estimation problems of chemical or nuclear power plants. However, the accuracies of traditional methods including Bayesian inference and optimization largely depend on the error of the model input and the accuracy of the forward dispersion modelling that is used in backward calculation. Furthermore, some input parameters such as atmospheric stability are quite difficult to measure and quantify. Therefore, methods such as using pre-determined scenarios for decisionmaking and bypassing the hard-to-obtain parameters have been proposed by researchers. ${ }^{25-28}$ These methods are able to estimate the source using neural networks or support vector machines without input data of certain complex parameters because a large number of pre-determined scenarios have already been applied for training and fitting. For example, a previous study estimated the release rate of a dispersion source via an ANN and optical sensor. ${ }^{28}$ Wang et al. bypassed the source term and used the integration of an ANN, gas detectors and PHAST to predict gas dispersion. ${ }^{27}$ The high accuracy of that study demonstrates that ANNs could be a useful tool for pollution forecasting and risk analysis. Moreover, an ANN was also applied for forecasting $\mathrm{PM}_{2.5}$ pollution and atmospheric dispersion of biological matter. ${ }^{29,30}$ Although ANNs have been extensively used in dispersion prediction, few researchers have applied an ANN in source location or quantification.

In this paper, a new approach is presented that is able to find the optimal emission source from candidate solutions using the measured data from a UAV. In order to generate high-quality training data for the ANN, an atmospheric dispersion simulation tool is used because the training input data are impossible to control in a real chemical industry park. The simulation tool uses the ANN to correct the traditional Gaussian diffusion model. The approach is then verified by numerical and real experiments. This approach can address the difficulties caused by the use of complex meteorological parameters as inputs. It is extremely insensible to measurement noise. Furthermore, the features of the ANN also make this novel approach more accurate than traditional methods. The experimental results show that the proposed source estimation method based on an ANN and a UAV is a useful and appropriate option for the management of a chemical industry park.

\section{Methods}

\subsection{Remote monitoring system based on UAV platform}

Conventional source estimation methods usually use concentration data from static monitoring stations. Therefore, the accuracy of the estimation results largely depends on the distribution and the number of monitoring stations. Generally, the more extensively and densely the monitoring stations are distributed, the more possible it is to identify the source correctly. Insufficient monitoring stations may cause large errors, especially when the contaminant plume does not pass directly over enough sensors. Measurements sampled near the central axis of the plume are more important because they provide more information on the features of the plume. We denote the measurements taken near the central axis "dominant data". This study uses a remote monitoring system based on a UAV platform to obtain the dominant data together with the corresponding location information with the aim of overcoming the disadvantages of static monitoring stations. This monitoring system is highly mobile, which significantly increases the probability of successfully finding the emission source.

2.1.1 Structure. The monitoring system uses MATRICE 100 produced by DJI as a UAV platform. ${ }^{31}$ As shown in Fig. 1, MATRICE 100 has four axes. The maximum takeoff weight is $3400 \mathrm{~g}$. The maximum flight duration is 20 minutes if gas sensors are installed and the aircraft has a single TB48D battery. If two batteries are installed, the flight duration can be extended to nearly 35 minutes. The maximum speed is $22 \mathrm{~m} \mathrm{~s}^{-1}$ and the cruising speed is $10 \mathrm{~m} \mathrm{~s}^{-1}$, which means this aircraft can travel approximately $20 \mathrm{~km}$ without changing batteries. This distance can easily meet the requirements of a typical industry park. Moreover, this aircraft is able to hover and automatically avoid collision. As shown in Fig. 1, on MATRICE 100, a microcomputer (Raspberry Pi $2 \mathrm{~b}$ ) is used for receiving and sending measurements. ${ }^{32}$ Because the flight duration is not long, the Raspberry Pi is powered by an independent battery to ensure uninterrupted sampling. In addition, the Raspberry Pi receives measurements from the gas sensors using a serial protocol. Currently, the gas monitoring system supports $\mathrm{O}_{2}, \mathrm{CO}_{2}, \mathrm{O}_{3}, \mathrm{H}_{2} \mathrm{~S}$, $\mathrm{SO}_{2}, \mathrm{CO}, \mathrm{NH}_{3}, \mathrm{NO}_{2}, \mathrm{C}_{2} \mathrm{H}_{4}$, benzene, methylbenzene, $\mathrm{PM}_{2.5}$, and $\mathrm{PM}_{10}$. In terms of data transmission, the aircraft sends measurements to a cloud database via an LTE transmitter.

2.1.2 Flight route. Generally, in order to obtain highquality data, the flight route is determined by the following basic rules. At the beginning, the aircraft moves in a circuit around the experimental area. When a peak appears in the measurement line (which means that the aircraft has just moved through the contaminant plume), the aircraft moves back and forth to sample the concentration data once again. After turning around several times, sufficient high-quality data have been obtained and the aircraft resumes its path along the original route.

Furthermore, planning a flight route is a complex task because many conditions, such as the capacity of the battery, potential barriers to movement, electromagnetic interference 


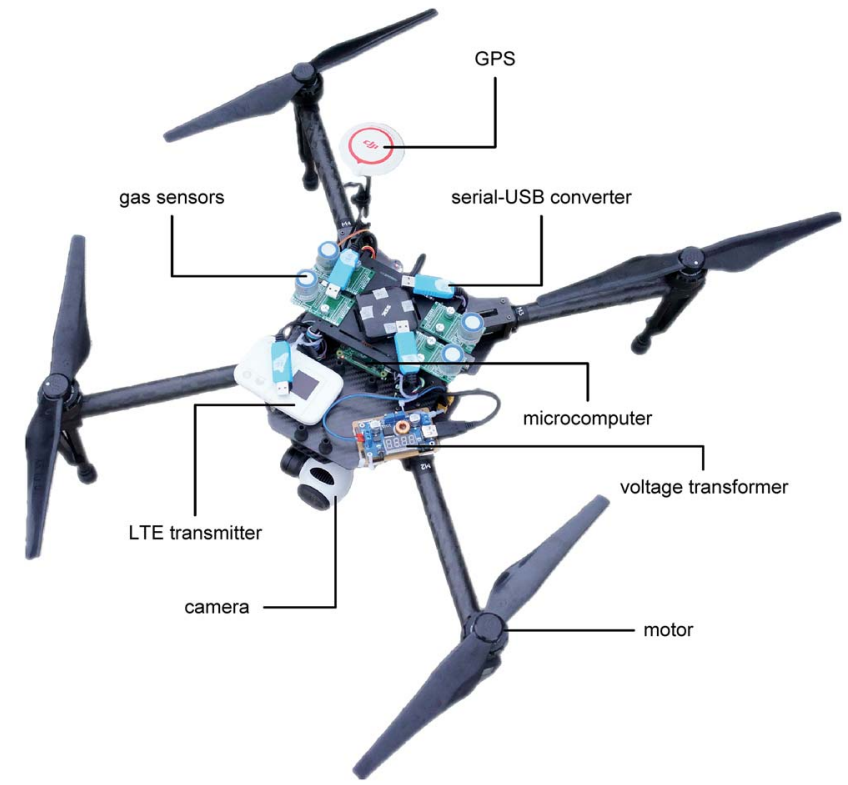

Fig. 1 Air concentration monitoring system based on UAV platform.

and the weather, must be considered. A well designed flight route can greatly improve the accuracy of source estimation. In this paper, we will not discuss the flight route planning in detail since the focus is the method of locating and quantifying the contaminant source.

\subsection{Source estimation using ANN}

Artificial neural networks can be used for fitting or classifying complex non-linear systems. Conventional source estimation methods are mostly based on Bayesian theory or optimization. They estimate the source term via calculating the posterior probability distribution or optimizing a cost function. These two methods are useful when no candidate solutions are available. However, in industry parks, it is already known which emission points are the possible sources of contaminants. Therefore, we can restrict the search for the real source to these potential emission points (candidate solutions), which means that the source location problem can be regarded as a classification problem. Highly accurate results can be obtained if the structure of the ANN is well designed.

In terms of traditional methods including Bayesian inference and optimization, it is clear that the inaccuracy in their results is mainly brought about by the errors in the input parameters. Clearly, some input parameters are impossible to directly measure using sensors. Therefore, to bypass these parameters, we use an atmospheric dispersion simulation tool to generate a sufficient number of pre-determined scenarios to cover all possible situations, and then use these scenarios to train the ANN. In Section 4, the experimental results show that the source estimation method based on the ANN is quite insensitive to these parameters.

2.2.1 Mechanism. The mechanism of the source estimation model is shown in Fig. 2. The complete set of measurements is fed as input to the source estimation model. The proposed approach firstly uses a convolution filter to eliminate noise. Then it selects the dominant data from the complete set of measurements. The position coordinates $(x, y, z)$ of the dominant data, the wind speed $v$ and the wind direction $d$ are fed as input to the ANN. The output of the ANN is the weight of each candidate. The release rate of the source can be calculated by optimizing a cost function.

The ANN used in this study is a typical neural network with a single hidden layer. The number of neurons in the hidden layer depends on the number of candidate sources. In terms of the input and output of the ANN, the input includes: (1) a threedimensional set $\{x, y, z\}$ whose elements are the $x$-axis coordinate $x, y$-axis coordinate $y, z$-axis coordinate $z$ of the sampling location of the dominant data; (2) wind speed $v$; and (3) wind direction $d$. In order to ensure the high quality of the sampling data and simplify the model, the aircraft usually moves at a constant height. In this case, we do not need to consider the parameter $z$. As for the output of the ANN, we denote the number of candidate sources $\left\{\theta_{i}=\left(x_{i}, y_{i}, z_{i}\right)\right\}_{i=1}^{N}$ as $N$. Therefore, the output is an $N$-dimensional set output $=\left\{w_{i}\right\}_{i=1}^{N}$ that represents the weight of all candidates. The $i^{\text {th }}$ element $w_{i}$ represents the weight of the candidate source $\theta_{i}$. A higher weight $w_{i}$ represents that the corresponding candidate $\theta_{i}$ is more likely to be the real emission source. If the real emission source is $\theta_{p}$ and the input is $\{x, y, z, d, v\}$, the expression of the output is shown as follows:

Output $=$

$\left\{w_{i} \mid w_{i}=\delta[i-p] \log _{10}[f(\theta, x, y, z, d, v)+1], i \in \mathrm{Z}, 1 \leq i \leq N\right\}$,

where $\delta[i]$ is the Dirac function and $f(\theta, x, y, z, d, v)$ is the atmospheric dispersion model for calculating the concentration, which will be explained in Section 2.3.2. Thus, the candidate source with the highest weight is the estimated contaminant source.

2.2.2 Source estimation. The process of source estimation is shown in Fig. 3. If the aircraft samples a total of $M$ measurements, all the sampled measurements can be written into the dataset $D=\left\{x_{t}, y_{t}, z_{t}, c_{t}\right\}_{t=1}^{M}$. The next step is to choose the dominant data from the dataset $D$. The basic principle of finding dominant data is to find the peaks in the concentration curve. However, noise in the concentration measurements may influence the results. To remove the effects of noise, a linear average filter is used to smooth the concentration curve by convolution manipulation. After the dataset has been smoothed, we use all peaks and their nearby measurements as dominant data $D_{c}=\left\{x_{\tau}, y_{\tau}, z_{\tau}, c_{\tau}\right\}_{\tau=1}^{M_{c}}$, where $M_{c}$ means the number of elements in $D_{c}$.

As a result, in the source estimation process, the input of the ANN is $\left\{d, v, x_{\tau}, y_{\tau}, z_{\tau} \mid\left\{x_{\tau}, y_{\tau}, z_{\tau}, c_{\tau}\right\} \in D_{c}\right\}_{\tau=1}^{M_{c}}$. Therefore, the output of the ANN is $\left\{w_{\tau, i}\right\}_{\tau=1, i=1}^{M_{c}, N}$, where $w_{\tau, i}$ means the weight of candidate source $\theta_{i}$ if the ANN input is $\left\{d, v, x_{\tau}, y_{\tau}, z_{\tau}\right\}$. However, each element in the input set may imply a different contaminant source. In order to address this problem, all the elements in the ANN output set are summed via the following equation: 


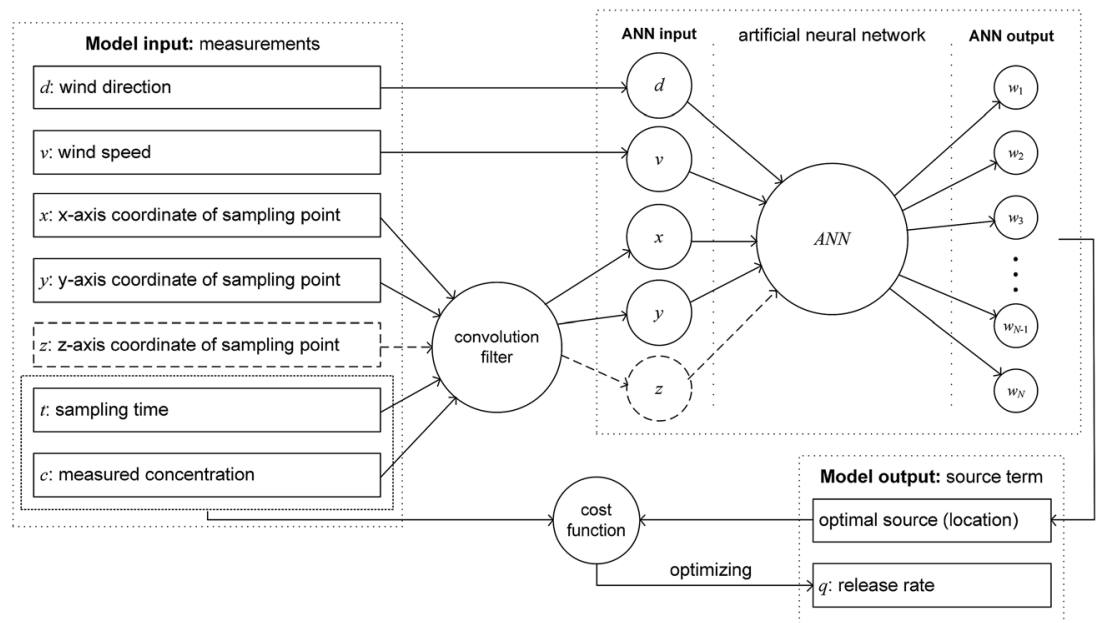

Fig. 2 Mechanism of source estimation model.

$$
W_{i}=\sum_{\tau=1}^{M_{c}} w_{\tau, i}
$$

where $W_{i}$ means the sum of weights $w_{i}$. Therefore, we can identify the optimal source as that which has the highest $W_{i}$. After finding the optimal source (denoted $\theta_{k}$ ), according to the maximum likelihood principle, its release rate $q_{k}$ can be estimated by optimizing (minimizing) the following cost function:

$$
J=\sum_{j=1}^{M}\left[q_{k} c_{k}\left(x_{j}, y_{j}, z_{j}\right)-c_{j}\right]^{2},\left\{x_{j}, y_{j}, z_{j}, c_{j}\right\} \in D
$$

This cost function can be solved by various methods such as the least squares algorithm and gradient descent. By solving it,

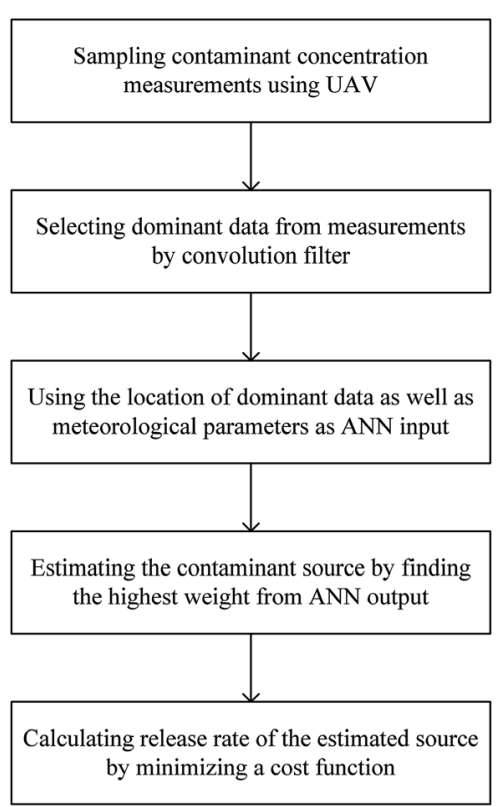

Fig. 3 Process of source estimation. the complete source term including location and release rate is successfully obtained.

\subsection{Contaminant atmospheric dispersion simulation and ANN training}

2.3.1 Scenario introduction. The experiments (both numerical and real) of this study are implemented in an industry park in Shanghai. A map of the analyzed area is illustrated in Fig. 4. In this figure, the origin point $O$ of the coordinate system is marked with the label "+". In these experiments, we investigate the emission of $\mathrm{SO}_{2}$ by measuring the $\mathrm{SO}_{2}$ concentrations. Five possible $\mathrm{SO}_{2}$ emission sources are located in this area. Therefore, we have five candidate sources. On the map, the 19 circles are the complete set of discharge points for all contaminants, among which the five orange circles are the $\mathrm{SO}_{2}$ discharge points. After projecting the WGS84 geographic coordinates into UTM Cartesian coordinates, the resulting positions of all the candidate sources are listed in Table 1 with some additional information. Yellow areas are the working area of the site; the blue area is the sea. The aircraft's usual flight path is over the safe areas marked in white or over the sea since unexpected crashes may cause serious damage to the chemical industry park. Choosing the optimal solution from five candidate sources appears to be a very simple problem. However, the flight duration of the UAV is limited, so the measured data are probably insufficient to support accurate calculation, especially when several candidates are in the same wind direction. Besides, for a specific kind of gas, it is quite common that the number of candidate sources is around five. Therefore, this scenario is meaningful for analyzing the source estimation method.

2.3.2 Virtual scenario generation. Both real data and simulation data can be used for training the ANN. However, if we use real data to train the ANN, it is necessary to install sensors across the entire chemical industry park. Additionally, the release rate of each candidate source must be controllable. It is quite difficult to meet these requirements, especially 


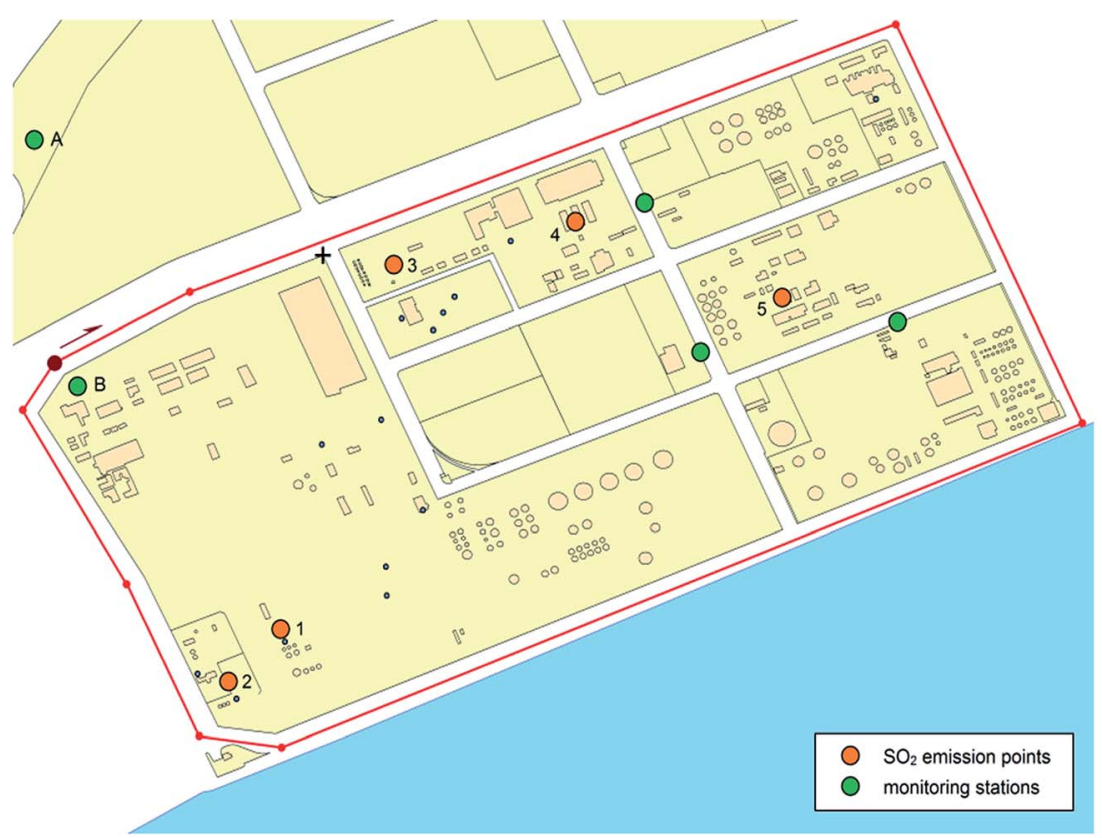

Fig. 4 Map of experimental area.

Table 1 Cartesian coordinates of $\mathrm{SO}_{2}$ emission points and additional information

\begin{tabular}{|c|c|c|c|c|c|}
\hline No. & $X$ & $Y$ & Height & Explanation & Contaminants \\
\hline 1 & -132.575 & -1317.63 & 50 & Waste incinerator for acrylonitrile (AN) & $\mathrm{SO}_{2}, \mathrm{NO}_{x}, \mathrm{VOC}, \mathrm{NH}_{3}$ \\
\hline 2 & -302.901 & -1483.42 & 68 & $\begin{array}{l}\text { Chimney of sulfuric acid recovery (SAR) } \\
\text { system }\end{array}$ & $\mathrm{SO}_{2}, \mathrm{NO}_{x}$, vitriol fog \\
\hline 3 & 267.1415 & 0.359916 & 27 & Furnace no. 1 & $\mathrm{SO}_{2}, \mathrm{PM} 2.5 / \mathrm{PM} 10$ \\
\hline 4 & 861.3643 & 147.0462 & 27 & Furnace no. 2 & $\mathrm{SO}_{2}, \mathrm{PM} 2.5 / \mathrm{PM} 10$ \\
\hline 5 & 1532.017 & -142.542 & 30 & Hazardous waste incinerator & $\begin{array}{l}\mathrm{CO}, \mathrm{SO}_{2}, \mathrm{NO}_{x}, \mathrm{PM} 2.5 / \mathrm{PM} 10, \mathrm{HF}, \mathrm{HCl}, \\
\text { dioxin }\end{array}$ \\
\hline
\end{tabular}

controlling the release rate. Thus, we use simulation scenarios to generate sufficient training data.

To simulate a contaminant dispersion scenario, several factors must be considered: emission source $\theta_{i}$, meteorological parameters (wind direction $d$ and wind speed $v$ ) and environmental conditions (atmospheric stability and terrain type over which the gas diffuses).

KD-ADSS is applied to model atmospheric dispersion. ${ }^{24} \mathrm{KD}-$ ADSS is an atmospheric dispersion simulation tool based on neural networks and a Gaussian diffusion model. It uses neural networks to calibrate the accuracy of the traditional Gaussian model. From the input parameters shown in Table 2 it generates an output, which is the concentration value at the point of interest. This simulation tool has also been validated by the commercial software PHAST, the Indianapolis field study and a study of the Fukushima Dai-ichi nuclear accident. Thus, the concentration at sampling point $(x, y, z)$ can be calculated by this simulation tool. The calculation of concentration data at each sampling point in the simulation can be illustrated by the function $f(\theta, x, y, z, d, v)$.

For the Gaussian diffusion coefficients $\sigma_{y}$ and $\sigma_{z}$, their expressions are: ${ }^{33}$

$$
\left\{\begin{array}{l}
\sigma_{y}\left(D_{x}\right)=a_{y} D_{x}\left(1+b_{y} D_{x}\right)^{-c_{y}} \\
\sigma_{z}\left(D_{x}\right)=a_{z} D_{x}\left(1+b_{z} D_{x}\right)^{-c_{z}}
\end{array},\right.
$$

where the parameters $a_{y}, b_{y}, c_{y}, a_{z}, b_{z}$, and $c_{z}$ depend on the environmental conditions (atmospheric stability and terrain

Table 2 Input parameters of atmospheric dispersion simulation tool

\begin{tabular}{ll}
\hline Input symbol & Meaning \\
\hline$q$ & Release rate of emission source \\
$W$ & $\begin{array}{l}\text { Wind field. KD-ADSS also contains a wind field } \\
\text { generation tool. }\end{array}$ \\
$H$ & Height of emission source \\
$D_{x}$ & Downwind distance of the interest point \\
$D_{y}$ & Crosswind distance of the interest point \\
$z$ & Height of the interest point \\
$\sigma_{y}$ & Gaussian diffusion coefficient of $y$-axis \\
$\sigma_{z}$ & Gaussian diffusion coefficient of $z$-axis \\
$v_{\mathrm{s}}$ & Deposition/rise velocity \\
Other parameters & This case does not need these parameters
\end{tabular}
concerning radionuclide 
Table 3 Relationships between diffusion coefficients and atmospheric stability

\begin{tabular}{|c|c|c|c|c|c|c|c|}
\hline Terrain & Class of atmospheric stability & $a_{y}$ & $b_{y}$ & $c_{y}$ & $a_{z}$ & $b_{z}$ & $c_{z}$ \\
\hline \multirow[t]{3}{*}{ Urban } & $\mathrm{A}$ and $\mathrm{B}$ & 0.32 & 0.0004 & -0.5 & 0.24 & 0.001 & -0.5 \\
\hline & $\mathrm{C}$ & 0.22 & 0.0004 & -0.5 & 0.2 & 0 & -0.5 \\
\hline & $\mathrm{E}$ and $\mathrm{F}$ & 0.11 & 0.0004 & -0.5 & 0.08 & 0.0015 & -0.5 \\
\hline \multirow[t]{4}{*}{ Open country } & A & 0.22 & 0.0001 & -0.5 & 0.2 & 0 & -0.5 \\
\hline & $\mathrm{B}$ & 0.16 & 0.0001 & -0.5 & 0.12 & 0 & -0.5 \\
\hline & $\mathrm{E}$ & 0.06 & 0.0001 & -0.5 & 0.03 & 0.0003 & -1 \\
\hline & $\mathrm{F}$ & 0.04 & 0.0001 & -0.5 & 0.16 & 0.0003 & -1 \\
\hline
\end{tabular}

type, as shown in Table 3). The training scenarios should cover as many environmental conditions as possible.

2.3.3 ANN training. It is usually difficult to obtain sufficient real data to train a neural network because controlling the release rate of each emission point and sampling across the entire region are not easy. Therefore, for each scenario, an atmospheric dispersion simulation tool is applied to model the contaminant dispersion and generate training data.

The training data of the ANN are generated by following workflow:

(1) Define the range of the training area (ranges of $x, y$ and $z$ );

(2) Define all possible atmospheric stabilities and terrains;

(3) Define the range of the ANN input parameters;

(4) Randomly generate sampling points in the training area;

(5) Use atmospheric dispersion models to calculate the ANN output;

(6) Generate input and target data for training and validation sets.

In each scenario, as can be seen in Table 4 , the release rates $q$ of all possible emission sources vary from 0 to $5 \mathrm{~g} \mathrm{~s}^{-1}$. The wind speed $v$ varies from 0 to $5 \mathrm{~m} \mathrm{~s}^{-1}$. The wind direction satisfies that $d \in\{x \mid 0<x \leq 360, x \in Z\}$. In order to cover a wider range of

Table 4 Training parameters for neural networks

\begin{tabular}{lll}
\hline Parameter & Explanation & Value \\
\hline$d$ & Wind direction (degree) & $1-360$ with step 1 \\
$v$ & Wind speed $\left(\mathrm{m} \mathrm{s}^{-1}\right)$ & $1-5$ with step 2 \\
$q$ & Release rate $\left(\mathrm{g} \mathrm{s}^{-1}\right)$ & $1-5$ with step 2 \\
$a_{y}, b_{y}, c_{y}$ & Parameters of $\sigma_{y}$ & See Table 3 \\
$a_{z}, b_{z}, c_{z}$ & Parameters of $\sigma_{z}$ & See Table 3 \\
$x_{\min }$ & Lower bound of & -2000 \\
& training area $x$-axis $(\mathrm{m})$ & \\
$x_{\max }$ & Upper bound of & 2000 \\
& training area $x$-axis $(\mathrm{m})$ & \\
$y_{\min }$ & Lower bound of & -1000 \\
& training area $y$-axis (m) & \\
$y_{\max }$ & Upper bound of & 3000 \\
$h$ & training area $y$-axis (m) & \\
$T_{\mathrm{s}}$ & Flight altitude (m) & 50 \\
& Number of sampling & 100 \\
& point for each release &
\end{tabular}

environmental conditions, ten different typical combinations of diffusion coefficients are selected to simulate the dispersion process (shown in Table 3). Table 3 indicates the relationship between diffusion coefficients and atmospheric stability according to Carrascal et $a .^{33}$ After using these scenarios to simulate the atmospheric dispersion process, we can then obtain the numerical concentrations measured at sampling points randomly distributed in $\left[x_{\min }, x_{\max }\right]$ and $\left[y_{\min }, y_{\max }\right]$. Moreover, we assume that the UAV platform moves at a constant height $h$ and the number of sampling points is $T_{\mathrm{s}}$. According to Section 2.2.1, we have four or five input ANN parameters. Because the flight height is a constant value $(50 \mathrm{~m})$, it is not necessary to consider the input parameter $z$ in Fig. 2. Therefore,

\section{(a) training set}

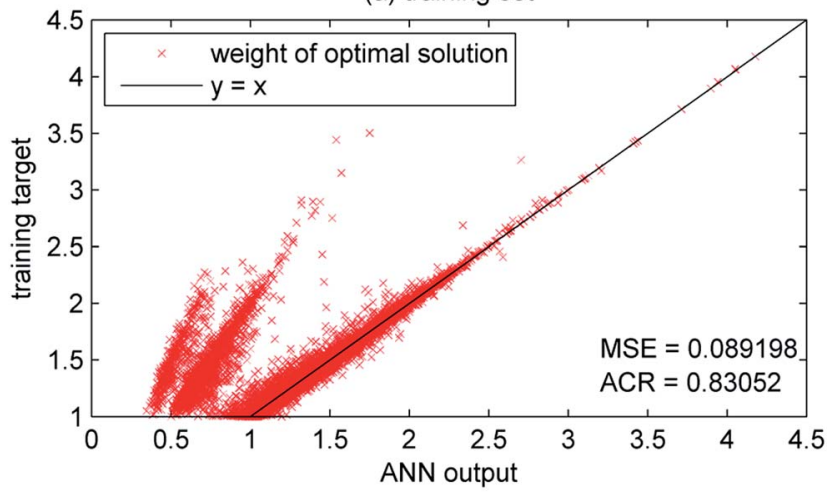

(b) validation set

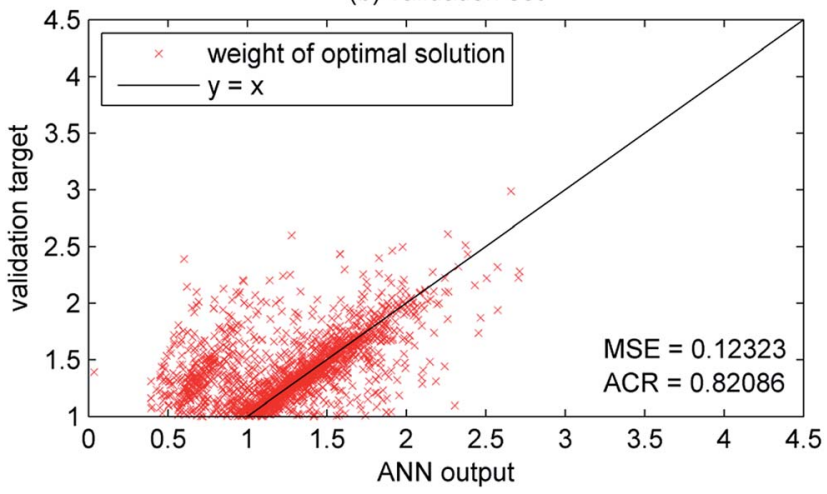

Fig. 5 Comparison of ANN output and training/validation target. 
the input layer of the ANN has four neurons. Furthermore, the number of neurons in the output layer equals the number of candidate sources since it is a classification ANN. It can be seen that there are only five candidate $\mathrm{SO}_{2}$ emission sources in Table 1 , so the output layer has five neurons. Because the detailed training procedure is beyond the research scope of this paper, the ANN will be directly trained by the MATLAB neural network toolbox. $^{34}$

As shown in Table 4 , we have $360 \times 3 \times 3 \times 10=32400$ different virtual scenarios. Since each scenario has 100 sampling points, we can obtain $32400 \times 100=3240000$ sets of data, $75 \%$ of which are for training and $25 \%$ of which are for validation. The average correct rate (ACR) of the training data is $83.05 \%$ and that of the validation data is $82.09 \%$. Furthermore, the comparison between the ANN output and training/ validation results is shown in Fig. 5. As can be seen from the figure, the ANN output (weight of optimal source) closely matches the training targets and validation targets, the mean square errors (MSE) of which are 0.089 and 0.123 respectively.

\section{Experimental}

\subsection{Numerical experiment}

A simulated numerical experiment is quite appropriate for testing the performances of most features of the proposed approach, because it is easier to set the parameters or variables in numerical experiments. In the numerical experiment, we analyse the performance of the ANN based source estimation method. Furthermore, the source estimation methods based on Bayesian inference and optimization are also used for comparison.

The source estimation method based on Bayesian inference is quite simple in this experiment because there are only five candidate sources. Thus, it is unnecessary to use a posterior distribution sampling algorithm (such as MCMC) in this experiment. After calculating the posterior probabilities of the five potential sources, the candidate source with the highest posterior probability is considered as the optimal solution.

In the optimization method, the cost function is defined as follows:

$\mathcal{J}_{k}=\lambda q_{k}^{2}+\sum_{j=1}^{M}\left[q_{k} c_{k}\left(x_{j}, y_{j}, z_{j}\right)-c_{j}\right]^{2},\left\{x_{j}, y_{j}, z_{j}, c_{j}\right\} \in D, 1 \leq k \leq 5$

where $\lambda$ is the penalty term to avoid the over-fitting problem. Each candidate source has a cost function $\mathcal{J}_{k}$. These cost functions can be minimized by Tikhonov regularization through adjusting the variable $q_{k}$. The candidate source with the minimum value of the cost function is the estimated emission source.

The numerical experiment requires these three methods to estimate the $\mathrm{SO}_{2}$ emission source using concentration data generated by the KD-ADSS simulation tool. In order to test the accuracy and stability of the source estimation approaches, the wind direction varies from 0 to $360^{\circ}$ for all test cases. The wind speed is $3.7 \mathrm{~m} \mathrm{~s}^{-1}$ and the release rate is $4.1 \mathrm{~g} \mathrm{~s}^{-1}$. To test the
Table 5 Control variables of numerical experiment

\begin{tabular}{ll}
\hline Control variable & Range \\
\hline Wind direction $d$ & 0 to $360(\mathrm{deg})$ \\
Concentration noise coefficient $f_{c}$ & 0 to 0.5 \\
Wind direction noise coefficient (deviation) $\sigma_{d}$ & 0 to $30(\mathrm{deg})$ \\
Atmospheric stability noise coefficient $f_{p}$ & 1 to 4
\end{tabular}

effect of environmental conditions, the diffusion coefficients $\sigma_{y}$ and $\sigma_{z}$ also vary during the test. Their expressions are as follows:

$$
\left\{\begin{array}{l}
\sigma_{y}\left(D_{x}\right)=f_{p} a_{y} D_{x} \cdot\left(1+b_{y} D_{x}\right)^{-c_{y}} \\
\sigma_{z}\left(D_{x}\right)=f_{p} a_{z} D_{x} \cdot\left(1+b_{z} D_{x}\right)^{-c_{z}}
\end{array},\right.
$$

where the atmospheric stability coefficient $f_{p}$ increases from 1 to 4 with steps of 0.06 to simulate different environmental conditions. Furthermore, the initial atmospheric stability corresponds to class $\mathrm{D}$, i.e. open country. Five scenarios are simulated, and in each scenario a different candidate source from among the five listed in Table 1 is the source of $\mathrm{SO}_{2}$.

Noise sources are added into the concentration measurements, wind direction, and diffusion coefficient in each test case to test the stability of the proposed method. The noise sources follow Gaussian distributions $N\left(0, \sigma^{2}\right)$. For the concentration noise $e_{c}$, the equation of its deviation $\sigma_{c}$ is $\sigma_{c}=f_{c} c$, where $c$ is the value of the measured concentration and $f_{c}$ is the noise coefficient, which increases from 0 to 0.5 . For the wind direction noise $e_{d}$, its deviation $\sigma_{d}$ increases from 0 to 30 . The noise of the diffusion coefficient follows a simple Gaussian distribution whose deviation is $0.1 \sigma_{x}$ or $0.1 \sigma_{y}$. All the control variables in the numerical experiment are shown in Table 5.

The flight route of the UAV is shown in Fig. 4, in which the moving velocity remains steady at $10 \mathrm{~m} \mathrm{~s}^{-1}$. The sensors sample the concentration data once per second. After using the simulation tool to calculate the $\mathrm{SO}_{2}$ concentrations of each scenario, the neural network, Bayesian inference and optimization methods are applied to test their performances.

\subsection{Real experiment}

After obtaining permission from the committee of the chemical industry park, a real experiment was conducted on $27^{\text {th }}$ May, 2016 in Shanghai. This experiment used ZE-03 $\mathrm{SO}_{2}$ sensors produced by Weisheng Inc. This real experiment was conducted in the same location introduced in Section 2.3. The aircraft took off and moved around the chemical industry park. When the aircraft arrived at the south-west corner, we found that the concentration of $\mathrm{SO}_{2}$ increased rapidly and soon reached its peak. At this location, the aircraft was manually controlled to turn around through the $\mathrm{SO}_{2}$ plume several times to sample sufficient data. According to our observation and investigation, the chimney of the sulfuric acid recovery (SAR) system was the only location from which $\mathrm{SO}_{2}$ gas was emitted during this time. As a result, we could be sure that the chimney of the SAR system was the emission source that caused the increase of the $\mathrm{SO}_{2}$ concentration. In addition, noise was added to the concentration and wind data to test the stability of the models. 


\section{Results and discussions}

\subsection{Results of numerical experiment}

The source estimation approach is applied in each test case of the numerical experiment and the results are presented in Fig. 6. These figures show the ACR of the ANN, Bayesian inference (maximum posterior probability) and optimization (minimum cost function). Fig. 6(a) shows the relationship between the ACR and wind direction $d$ when $d$ increases from 0 to $360^{\circ}$. The other parameters are: $f_{\mathrm{c}}=0.1, \sigma_{d}=5$, and $f_{p}=1$. As can be seen in this figure, the ACR curves of the ANN, Bayesian inference and optimization have similar trends. The ACR of the ANN (77.38\%) is clearly higher than that of Bayesian inference $(74.56 \%)$ and optimization $(72.17 \%)$. When the wind direction satisfies that $d \in(120,180) \cup(300,360)$, the correct rates of these three methods are quite high (nearly 100\%). However, when the wind direction $d$ is not in this range, the correct rates of ANN, Bayesian inference and optimization fluctuate between approximately $50-90 \%$, 40-90\% and $20-90 \%$ respectively. Therefore, the performance of the ANN is better than Bayesian inference and optimization. It is worth mentioning that some candidate sources may generate very similar measurements in some directions. Thus, the tested methods may therefore mistakenly identify the wrong source, which is also the reason for some obvious errors appearing in the ANN training results in Fig. 5. To address this problem, turning the aircraft around several times to sample more data or re-designing a more effective flight route may improve the results.

Fig. 6(b) shows the effect of the noise deviation of wind direction $\sigma_{d}$ when $d=0, f_{c}=0.1$ and $f_{p}=1$. As we can see in this figure, when $\sigma_{d}$ is less than 5 , the correct rates of Bayesian inference and optimization remain stable at very high accuracy, while that of the ANN experiences a slight decrease. $\sigma_{d}$ when $\sigma_{d}$ is larger than 5, the ACR of the ANN becomes higher than the other two methods. All three methods drop to around $40 \%$ with the maximum noise coefficient. Therefore, all three methods are relatively sensitive when noise is added into the wind direction. Consequently, the accuracy of source estimation heavily depends on the accuracy of the meteorological parameters.

Fig. 6(c) illustrates the ACR as a function of the concentration noise coefficient $f_{\mathrm{c}}$ when $d=0, \sigma_{d}=5$ and $f_{p}=1$. Clearly, the concentration noise has almost no influence on the accuracies of all three methods. The correct rates of the ANN, Bayesian inference and optimization remain stable at around $90 \%$. Therefore, all three source estimation methods are quite stable when noise is added into the concentration data.

In terms of the atmospheric stability coefficient $f_{p}$, Fig. 6(d) indicates the relationship between $f_{p}$ and the accuracy when $d=$ $0, f_{c}=1$, and $\sigma_{d}=5$. The purpose of this case is to test the stability of the source estimation approaches when the environmental conditions (especially atmospheric stability) vary. In the atmospheric dispersion model, Gaussian diffusion coefficients are used for describing the atmosphere stability. Therefore, we use eqn (5) to set a series of test cases with different atmospheric stabilities. $f_{p}=1$ represents the atmospheric condition of class D in open country. $f_{p}=4$ represents that the atmosphere is extremely unstable. As we can see in Fig. 6(d), the estimation approach based on the ANN is quite robust when the atmospheric stability varies. However, the accuracy of Bayesian inference significantly decreases during this test, dropping to (a)

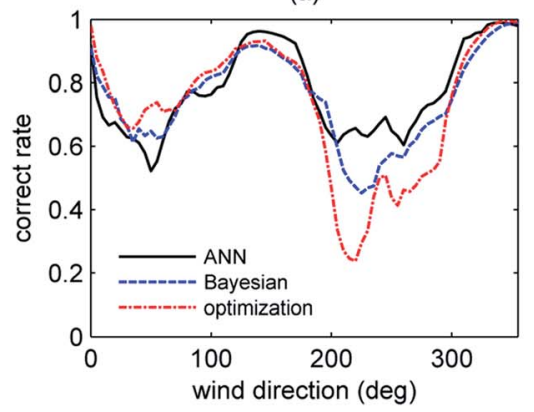

(c)

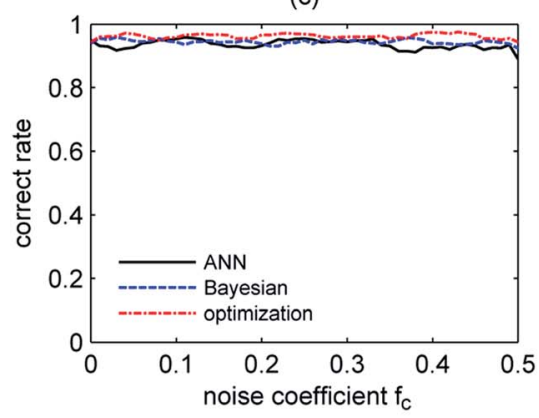

(b)

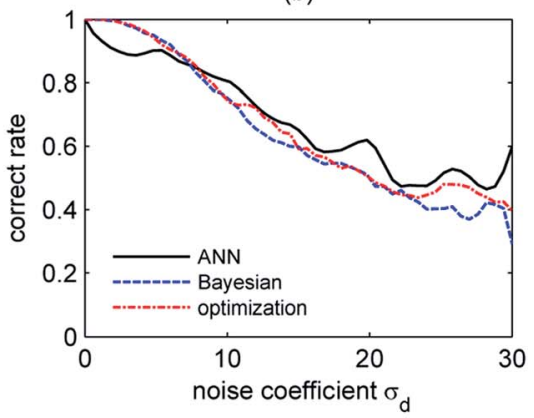

(d)

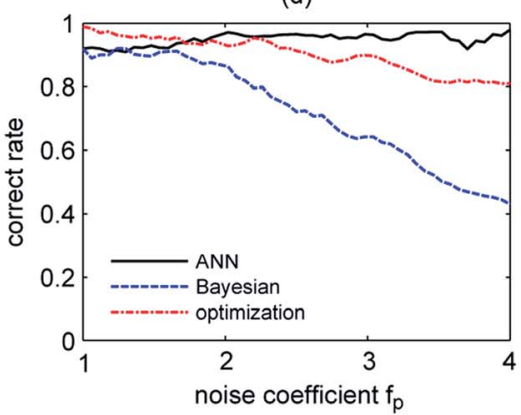

Fig. 6 Results of numerical experiment: (a) Effect of wind direction $d$. (b) Effect of noise deviation of wind direction $\sigma_{d}$. (c) Effect of noise coefficient of concentration $f_{c}$. (d) Effect of atmospheric stability coefficient $f_{p}$. 


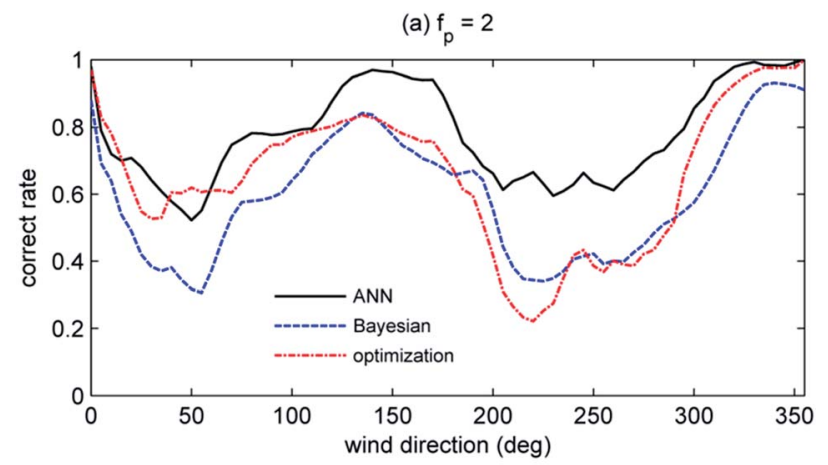

(b) $f_{p}=4$

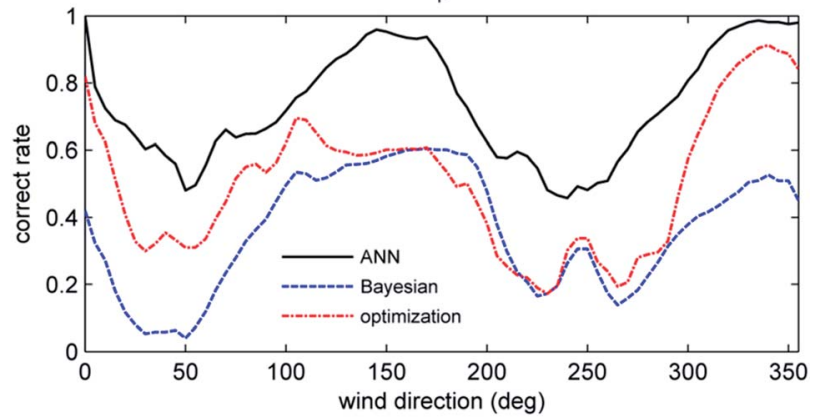

Fig. 7 Correct rate of ANN, Bayesian inference and optimization when (a) $f_{p}=2$ and (b) $f_{p}=4$.

only $40 \%$ when $f_{p}=4$. The optimization method also shows a downward trend, finally falling to $80 \%$.

Therefore, noise sources in both wind direction and concentration have similar effects on these three methods with the accuracy of the ANN being slightly higher. When it comes to atmospheric stability, the advantage of the ANN becomes much more obvious. The most important reason that we use the ANN is to bypass the effect of atmospheric stability. The experimental results show that the ANN can effectively meet our requirement.
To further analyze the features of the proposed approach, we test the performances of the source estimation methods as a function of atmospheric stability in all possible wind directions when $d=0, \sigma_{d}=5$ and $f_{c}=0.1$ (presented in Fig. 7). It is clear that the performance of the ANN remains good in different atmospheric stabilities at all times, while the accuracy of Bayesian inference becomes significantly lower if the atmosphere becomes unstable. Because of the penalty term in the cost function, the correct rate of optimization drops by at most $40 \%$ and its performance is slightly better than Bayesian inference.

Generally, the atmospheric stability is very difficult to quantify, while other parameters such as wind direction and concentration can be easily measured. Therefore, determining the precise atmospheric stability as well as the diffusion coefficient is a troubling issue in traditional methods like Bayesian inference and optimization. Fortunately, the source estimation approach based on an ANN can address this problem effectively, which makes it highly promising in real applications.

\subsection{Results of real experiment}

The measurements of the real experiment are shown in Fig. 8. When the aircraft arrived at the south-west corner, the measured concentration dramatically increased. Therefore, the aircraft was manually controlled to fly forwards and backwards over the south-west corner several times in order to sample sufficient dominant data. Fig. 8(a) represents the measured concentrations sampled by the UAV platform. As we can see in this figure, the aircraft spent most of its time flying around the south-west corner (data no. 300 to no. 1200) and the highest concentration reached almost $16 \mathrm{mg} \mathrm{m}^{-3}$.

Via the method introduced in Section 2.2, the original measurements are smoothed by a linear average filter. Thus, the dominant data are then selected according to the proposed method in Section 2.2. The smoothed $\mathrm{SO}_{2}$ concentration data
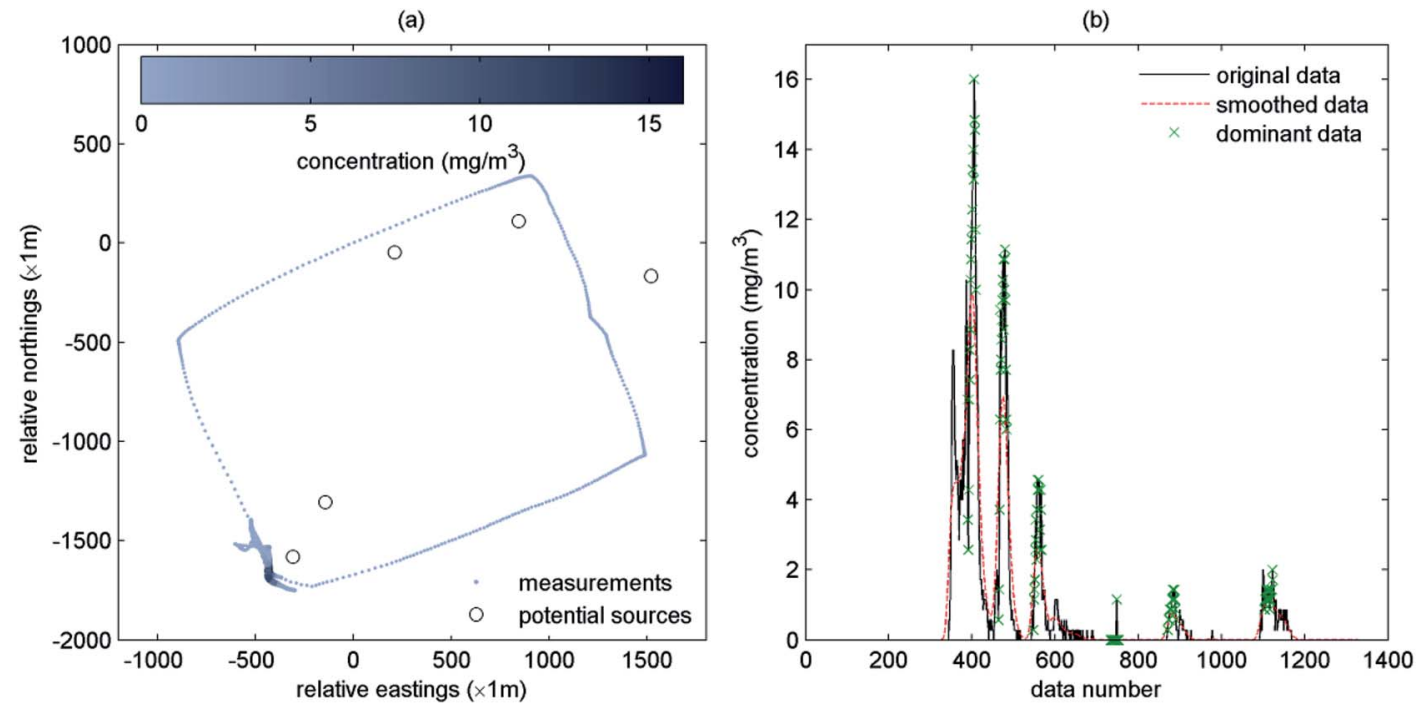

Fig. 8 The $\mathrm{SO}_{2}$ measurements of the real experiment: (a) Flight route and measured concentration are shown as a scatter plot. (b) Original concentration data, smoothed data, and selected dominant data. 
Table 6 Corresponding meteorological data of each dominant subset

\begin{tabular}{lll}
\hline $\begin{array}{l}\text { Dominant } \\
\text { subset ID }\end{array}$ & $\begin{array}{l}\text { Average wind } \\
\text { direction }(\mathrm{deg})\end{array}$ & $\begin{array}{l}\text { Average wind speed } \\
\left(\mathrm{m} \mathrm{s}^{-1}\right)\end{array}$ \\
\hline 1 & 81.7 & 1.9 \\
2 & 90.5 & 3.4 \\
3 & 85.3 & 4.2 \\
4 & 103.7 & 4.4 \\
5 & 105.5 & 1.7 \\
6 & 102.8 & 2.9
\end{tabular}

and dominant data are displayed in Fig. 8(b) together with the original data. According to Fig. 8(b), the dominant data contain six dominant subsets. The average wind directions and wind speeds of all dominant subsets are presented in Table 6 . Thus, the trained ANN is applied to calculate the output from these data (shown in Fig. 9). All of these input subsets result in the same conclusion - "chimney of SAR system" is the estimated emission source according to the sum of each output.
To test the sensitivity in real situations, virtual noises are added into the input variables to study the resulting uncertainty of the output. Because atmospheric stability is quite difficult to measure, we only test the sensitivity of the proposed method to changes in wind direction, wind speed and concentration. The virtual noises include wind direction noise $e_{d}$, wind speed noise $e_{v}$ and concentration noise $e_{c}$, following the normal distributions $e_{d} \sim N\left(0, \sigma_{d}\right), e_{v} \sim N\left(0, \sigma_{v}\right)$ and $e_{c} \sim N\left(0, f_{c} c\right)$ respectively. The effect of wind direction is shown in Fig. 10 when $\sigma_{v}=1$ and $f_{c}=$ 0.1. As can be seen from the figure, if the deviation of wind direction satisfies $\sigma_{d} \leq 12^{\circ}$, the accuracy is quite high and the ACR remains stable at almost $100 \%$. If $\sigma_{d}>12^{\circ}$, the ACR begins to decline gradually, decreasing to around $50 \%$ when $\sigma_{d}$ reaches $30^{\circ}$. We can therefore conclude that the stability of the proposed method with respect to wind direction is acceptable in practice. In terms of wind speed, the sensitivity analysis result shows that the ACR remains unchanged at $100 \%$ when the deviation of wind speed noise varies from 0 to $3 \mathrm{~m} \mathrm{~s}^{-1}$. For the uncertainty in concentration, we find it is unnecessary to plot its sensitivity analysis result because the ACR is always $100 \%$ when the noise
- waste incinerator for AN $\square \quad$ chimney of SAR system

(a)

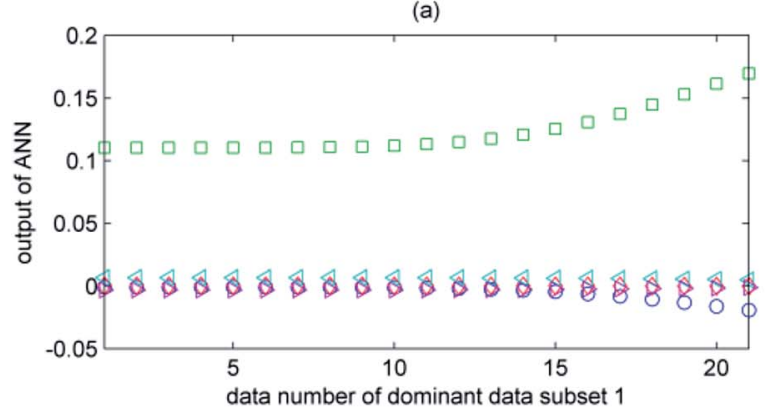

(c)

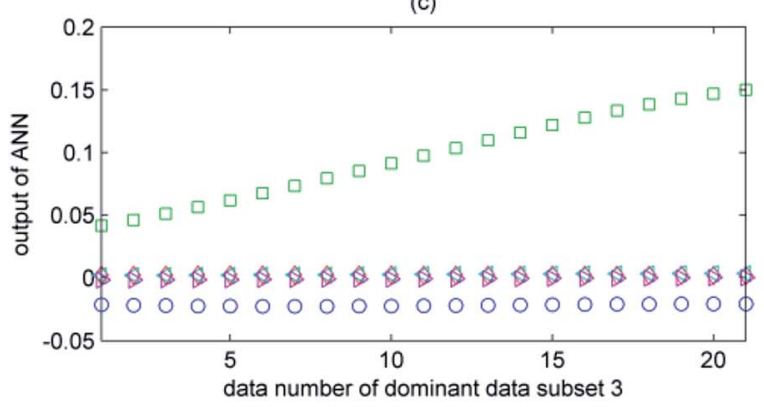

(e)

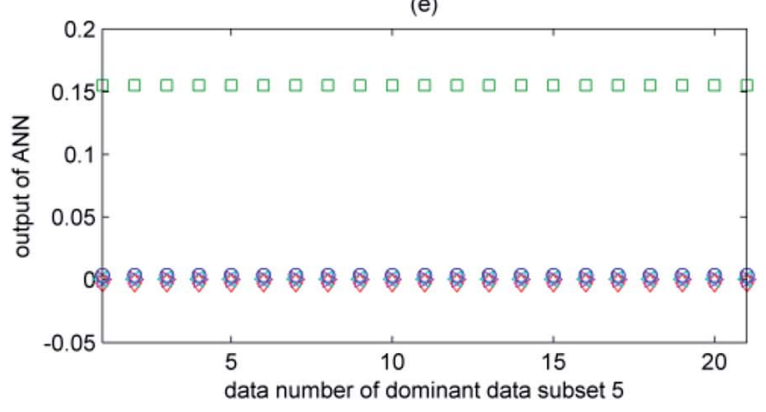

Fig. 9 The neural network output of six dominant subsets. furnace No. $1 \triangleleft$ furnace No. $2 \quad \triangleright \quad$ hazardous waste incinerator

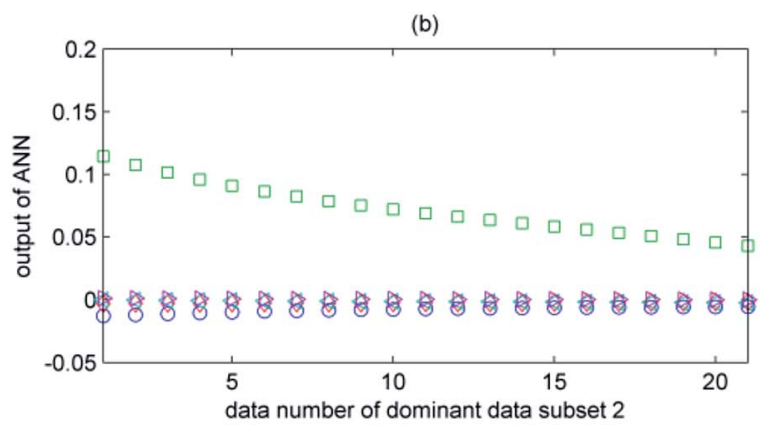

(d)

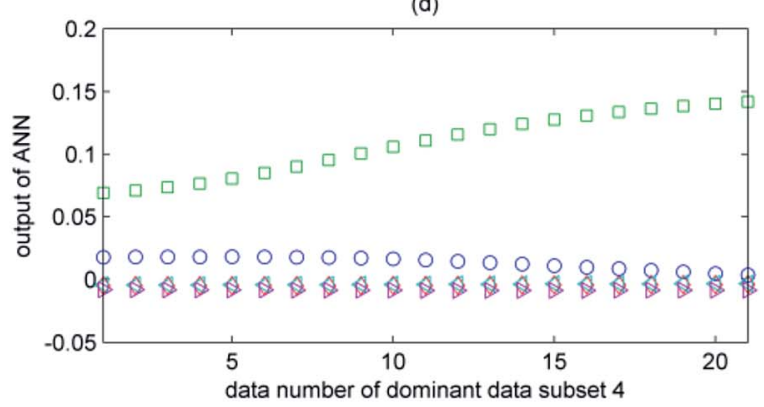

(f)

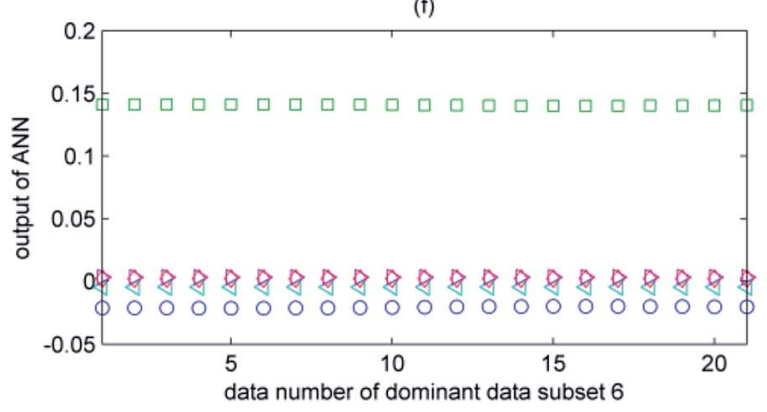




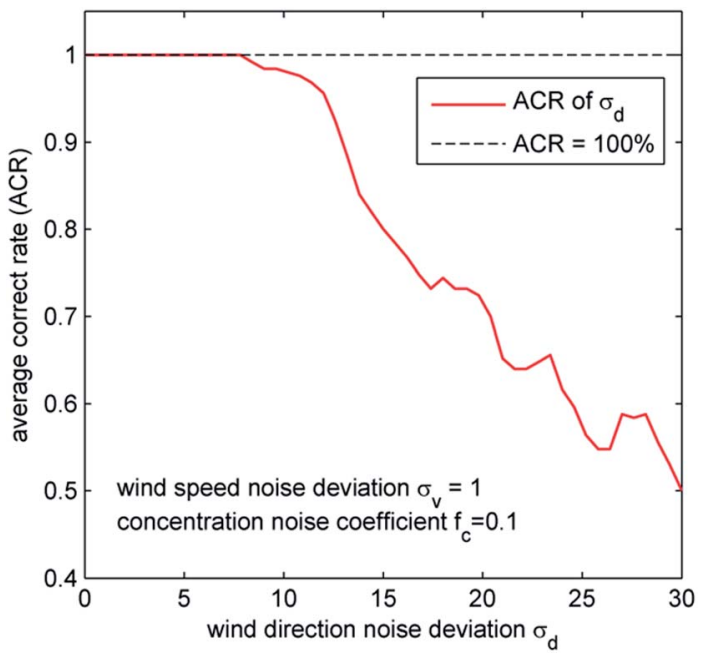

Fig. 10 Sensitivity analysis result of wind direction.

coefficient of concentration satisfies that $0 \leq f_{c} \leq 0.5$. In practice, $f_{c}=0.5$ is a fairly high concentration noise, which is not common in real sensors. Therefore, the proposed method is extremely insensitive to concentration noise. This confirms that the use of low-price sensors on the UAV platform is acceptable for source term estimation.

\section{Discussions}

As mentioned in Section 4.1, the accuracy of the source estimation methods may be low in some wind directions. For example, when wind direction $d=15^{\circ}$, the trends of the $\mathrm{SO}_{2}$ air concentration measurements generated by the waste incinerator for acrylonitrile, the chimney of the SAR system, and Furnace no. 1 are quite similar. All of them reach their

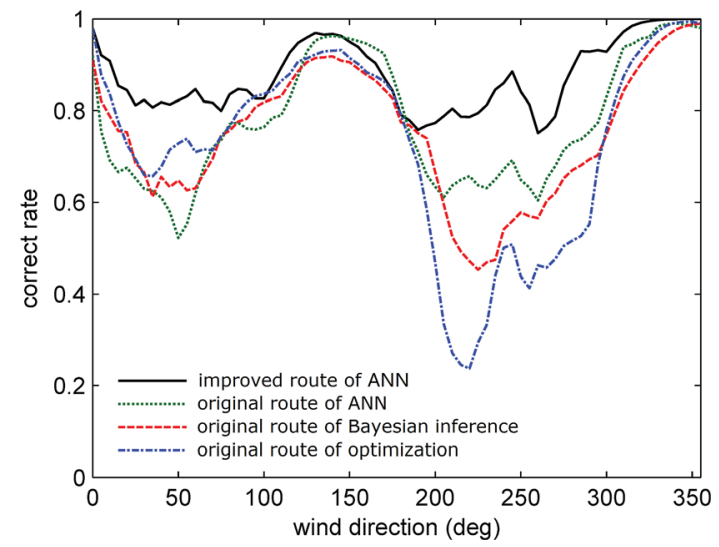

Fig. 12 Source estimation results of ANN, Bayesian inference, optimization and ANN with the improved route.

concentration peaks in the south-west corner. Because the $\mathrm{SO}_{2}$ gas plumes of these potential sources are overlapped at the south-west corner when $d=15^{\circ}$, it is difficult to identify exactly which of them emits the contaminant. To settle this problem, we suggest to re-plan the flight route to sample dominant data containing more useful information. An effective flight route can avoid ambiguity of identification. For example, Fig. 11 shows an alternative flight route of the UAV platform and Fig. 12 shows its identification results. Clearly, changing the original flight route can greatly improve the accuracy (the mean correct rate is $87.64 \%$ ).

However, planning the flight route is a complex task, and is not the focus of our current research work. Furthermore, the flight route also has some restrictions. For example, some facilities may be too dangerous for the aircraft to fly over them.

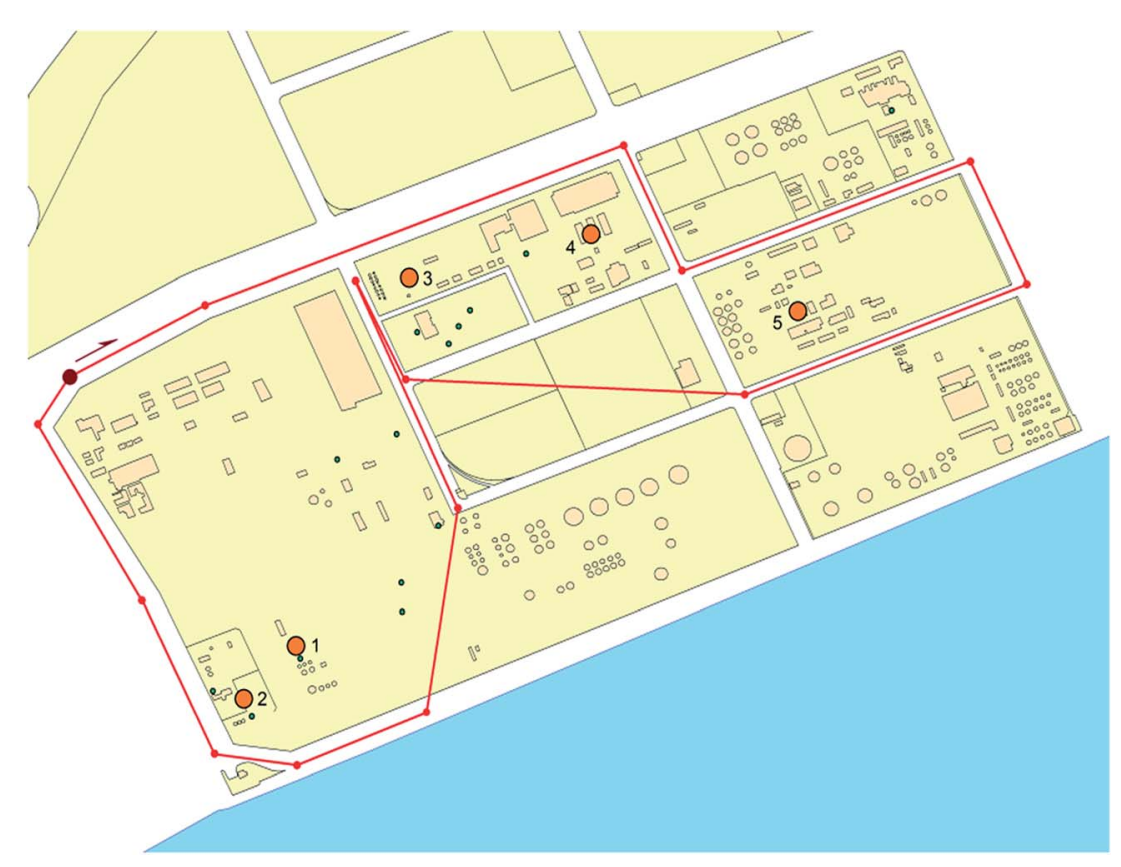

Fig. 11 An alternative, improved flight route for contaminant concentration sampling. 
Therefore, designing an effective flight route is an important but challenging task.

The numerical experiment compares the ANN-based source estimation approach with methods based on Bayesian inference and optimization. The source estimation approach based on the ANN is more stable in changing environmental conditions. The ACR of this approach demonstrates almost no change (as shown in Fig. 7) when the atmospheric stability coefficient is changed from 1 to 4 (stable to unstable). Atmospheric stability and other complex meteorological parameters that are used in Bayesian inference and optimization cannot be precisely measured. However, the ANN-based approach is able to bypass these parameters. In terms of the effect of wind direction, the ACR of the ANN-based approach $(77.38 \%)$ is higher than those of Bayesian inference and optimization $(74.56 \%$ and $72.17 \%$ respectively) because it has been trained on pre-determined scenarios. In the training set, the ACR of the ANN is $83.05 \%$, and the ACR in the validation set is only slightly lower (82.08\%). A well-trained ANN is the basis of accurate inverse calculation, which makes the ANN-based approach the optimal option for source estimation in chemical industry parks. The weak point of the ANN is that the investigation area must be pre-determined, because the source estimation approach based on the ANN needs a large number of training data sampled from this area. Moreover, the number of candidate sources cannot be too large and the ANN cannot identify multiple sources, which are disadvantages that it shares with other traditional methods. However, in chemical industry parks, it is easy to obtain detailed information of the scenario and candidate sources, which means that the ANN method is quite suitable for daily monitoring and management of chemical industry parks.

The real experiment implemented the source estimation method using real $\mathrm{SO}_{2}$ concentration data. The proposed approach was also verified by the observed and investigated results. Because of the flexibility of the UAV platform, the remote monitoring system was able to sample data containing useful information. The experimental results show that the ACR concerning wind direction is quite high when the error deviation is less than around $12^{\circ}$. Furthermore, the presence of noise in the wind speed and concentration data has almost no influence on the accuracy: the ACR remains at $100 \%$ at all times when these noise sources vary within realistic ranges. Although the resolution and accuracy of the sensors installed on the UAV platform (ppb or ppm level) were much lower than those of static sensors (ppt or ppb level), the proposed method was acceptably insensitive to the measurement errors. Consequently, the real experiment illustrates the advantages of the source estimation approach based on an ANN and a UAV platform and demonstrates that this approach can be successfully applied in a real chemical industry park.

\section{Conclusion}

This paper proposed an approach for estimating a contaminant dispersion source based on a UAV platform, an ANN and an atmospheric dispersion simulation tool. Using a UAV platform can overcome the limitations of static sensors and allow the sampling of high-quality concentration data containing important spatial information. Using an ANN can bypass the need for complex parameters and mechanisms of backward calculation and provide stability when the environmental conditions change dramatically. Because this platform is insensitive to noise added into the concentration measurements, it is feasible to install gas sensors that are relatively inaccurate but lightweight. However, noise in the wind direction data can significantly influence the final accuracy. The meteorological data should therefore be of high accuracy and precision, in agreement with the conclusions of previous studies. Furthermore, planning an effective flight route is an important measure to promote the accuracy of source estimation.

The approach proposed in this paper had a positive impact in practice. A more advanced monitoring system is now under development. The gas sensors have been upgraded to the $\mu \mathrm{g}$ $\mathrm{m}^{-3}$ level. In addition, Raspberry Pi has been replaced by a stable micro-controller. In terms of the source estimation approach, the current version is only appropriate for singlesource identification. Therefore, a multi-source identification algorithm will be investigated in the future.

\section{Conflicts of interest}

There are no conflicts to declare.

\section{Acknowledgements}

This study is supported by National Key Research \& Development (R\&D) Plan of China under Grant No. 2017YFC0803300, the National Natural Science Foundation of China under Grant No. 71673292 \& 61503402, Guangdong Key Laboratory for Big Data Analysis and Simulation of Public Opinion and Shanghai Special Foundation of Software and Integrated Circuit under Grant No. 150312.

\section{References}

1 Y.-S. Chung, S.-H. Kim, J.-H. Moon, Y.-J. Kim, J.-M. Lim and J.-H. Lee, J. Radioanal. Nucl. Chem., 2006, 267, 35-48.

2 G. Katata, M. Chino, T. Kobayashi, H. Terada, M. Ota, H. Nagai, M. Kajino, R. Draxler, M. C. Hort, A. Malo, T. Torii and Y. Sanada, Atmos. Chem. Phys., 2015, 15, 10291070.

3 A. Keats, E. Yee and F.-S. Lien, Atmos. Environ., 2007, 41, 465479.

4 E. Lushi and J. M. Stockie, Atmos. Environ., 2010, 44, 10971107.

5 V. Winiarek, J. Vira, M. Bocquet, M. Sofiev and O. Saunier, Atmos. Environ., 2011, 45, 2944-2955.

6 M. Hutchinson, H. Oh and W.-H. Chen, Inform. Fusion, 2017, 36, 130-148.

7 N. A. Gatsonis, M. A. Demetriou and T. Egorova, 2015 IEEE International Symposium on Technologies for Homeland Security (HST), 2015, pp. 1-6. 
8 B. Hirst, P. Jonathan, F. González del Cueto, D. Randell and O. Kosut, Atmos. Environ., 2013, 74, 141-158.

9 M. L. Melamed, S. Solomon, J. S. Daniel, A. O. Langford, R. W. Portmann, T. B. Ryerson, J. D. K. Nicks and S. A. McKeen, J. Environ. Monit., 2003, 5, 29-34.

10 T. Mori, T. Hashimoto, A. Terada, M. Yoshimoto, R. Kazahaya, H. Shinohara and R. Tanaka, Earth, Planets Space, 2016, 68, 1-18.

11 Y. Sanada and T. Torii, J. Environ. Radioact., 2015, 139, 294299.

12 A. Sinha, A. Tsourdos and B. White, IFAC Proceedings Volumes, 2009, 42, 7-12.

13 B. White, A. Tsourdos, I. Ashokaraj, S. Subchan and R. Zbikowski, in AIAA Guidance, Navigation and Control Conference and Exhibit, American Institute of Aeronautics and Astronautics, 2007, DOI: 10.2514/6.2007-6761.

14 Z.-R. Peng, D. Wang, Z. Wang, Y. Gao and S. Lu, Atmos. Environ., 2015, 123, 357-369.

15 S. K. Rao, Atmos. Environ., 2007, 41, 6964-6973.

16 M. Borysiewicz, A. Wawrzynczak and P. Kopka, Foundations of Computing and Decision Sciences, 2012, 37, 253.

$17 \mathrm{E}$. Yee, presented in part at the Chemical and Biological Sensing VIII, ed. W. Fountain Augustus III, Proceedings of the SPIE, 2007.

18 L. Tierney, Ann. Statist., 1994, 22, 1701-1728.

19 T. M. Chin and A. J. Mariano, J. Atmos. Ocean. Tech., 2010, 27, 371-384.

20 A. Wawrzynczak, P. Kopka and M. Borysiewicz, presented in part at the Parallel Processing and Applied Mathematics: 10th International Conference, Berlin, Heidelberg, 2014, pp. 407417.
21 Y. Zhang and L. Wang, Advanced Technology in Teaching: Selected papers from the 2012 International Conference on Teaching and Computational Science (ICTCS 2012), 2013, pp. 517-523, DOI: 10.1007/978-3-642-29458-7_76.

22 X. L. Zhang, G. F. Su, H. Y. Yuan, J. G. Chen and Q. Y. Huang, J. Hazard. Mater., 2014, 280, 143-155.

23 D. Ma, S. Wang and Z. Zhang, Atmos. Environ., 2014, 94, 637646.

24 S. Qiu, B. Chen, Z. Zhu, Y. Wang and X. Qiu, J. Radioanal. Nucl. Chem., 2016, 1-14, DOI: 10.1007/s10967-016-4941-z.

25 V. M. Krasnopolsky and H. Schiller, Neural Networks, 2003, 16, 321-334.

26 V. Vukovic, P. C. Tabares-Velasco and J. Srebric, J. Air Waste Manage. Assoc., 2010, 60, 1034-1048.

27 B. Wang, B. Chen and J. Zhao, J. Hazard. Mater., 2015, 300, 433-442.

28 W. So, J. Koo, D. Shin and E. S. Yoon, Comput.-Aided Chem. Eng., 2010, 28, 199-204.

29 K. Akahane, S. Yonai, S. Fukuda, N. Miyahara, H. Yasuda, K. Iwaoka, M. Matsumoto, A. Fukumura and M. Akashi, Environmentalist, 2012, 32, 136-143.

30 X. Feng, Q. Li, Y. Zhu, J. Hou, L. Jin and J. Wang, Atmos. Environ., 2015, 107, 118-128.

31 DJI. Inc., Matrice 100-Specifications, http://wiki.dji.com/en/ index.php/Matrice_100-Specifications, accessed 11 August, 2016.

32 Raspberry PI, Raspberry Pi 2 Model B, http:// www.raspberrypi.org/products/raspberry-pi-2-model-b/.

33 M. D. Carrascal, M. Puigcerver and P. Puig, Theor. Appl. Climatol., 1993, 48, 147-157.

34 MATLAB, Version 7.11.0, The MathWorks Inc., Natick, Massachusetts, 2010. 